\title{
Information Technology to Support Improved Care For Chronic Illness
}

\author{
Alexander S. Young, MD, MSHS ${ }^{1,2}$, Edmund Chaney, $P h D^{3,4}$, Rebecca Shoai, MPH, MSW', \\ Laura Bonner, PhD ${ }^{3}$, Amy N. Cohen, PhD ${ }^{7}$, Brad Doebbeling, $M D^{5,6}$, David Dorr, MD ${ }^{7,8}$, \\ Mary K. Goldstein, $M D^{9,10}$, Eve Kerr, MD, MPH 17,12, Paul Nichol, $M D^{3}$, and Ruth Perrin, $M A^{13}$
}

\begin{abstract}
'West Los Angeles VA Healthcare Center, VA Desert Pacific Mental Illness Research, Education and Clinical Center (MIRECC), Los Angeles, CA, USA; ${ }^{2}$ University of California, Los Angeles (UCLA), Los Angeles, CA, USA; ${ }^{3}$ VA VISN Health Services Research and Development Center, Seattle, WA, USA; ${ }^{4}$ University of Washington, Seattle, WA, USA; ${ }^{5}$ Indianapolis VA, Indianapolis, IN, USA; ${ }^{6}$ Indiana University School of Medicine, Indianapolis, IN, USA; ${ }^{7}$ Portland VA, Portland, OR, USA; ${ }^{8}$ Department of Medical Informatics and Clinical Epidemiology, Oregon Health and Science University, Portland, OR, USA; ${ }^{9}$ VA Palo Alto Health Care System, Geriatrics Research Education and Clinical Center (GRECC), Palo Alto, CA, USA; ${ }^{10}$ Stanford University, Stanford, CA, USA; ${ }^{11}$ Ann Arbor VA, Ann Arbor, MI, USA; ${ }^{12}$ University of Michigan, Ann Arbor, MI, USA; ${ }^{13}$ VA Information Resource Center (VIReC), Hines, IL, USA.
\end{abstract}

BACKGROUND: In populations with chronic illness, outcomes improve with the use of care models that integrate clinical information, evidence-based treatments, and proactive management of care. Health information technology is believed to be critical for efficient implementation of these chronic care models. Health care organizations have implemented information technologies, such as electronic medical records, to varying degrees. However, considerable uncertainty remains regarding the relative impact of specific informatics technologies on chronic illness care.

OBJECTIVE: To summarize knowledge and increase expert consensus regarding informatics components that support improvement in chronic illness care. Design: A systematic review of the literature was performed. "Use case" models were then developed, based on the literature review, and guidance from clinicians and national quality improvement projects. A national expert panel process was conducted to increase consensus regarding information system components that can be used to improve chronic illness care.

RESULTS: The expert panel agreed that informatics should be patient-centered, focused on improving outcomes, and provide support for illness self-management. They concurred that outcomes should be routinely assessed, provided to clinicians during the clinical

Electronic supplementary material Supplementary material is available for this article at doi:10.1007/s11606-007-0303-4.

This work was supported by the Department of Veterans Affairs through the Health Services Research and Development Service QUERI, (MHS-03218) and the Desert Pacific Mental Illness Research, Education and Clinical Center (MIRECC), and by the NIMH UCLA-RAND Center for Research on Quality in Managed Care (MH 068639). Any opinions expressed in this manuscript are the authors' and do not necessarily represent the views of the Department of Veterans Affairs.

An earlier version of this paper was presented at the Department of Veterans Affairs Research and Development State of the Art Conference, "Managing Complexity in Chronic Care," Arlington, Va, September 13-15, 2006. encounter, and used for population-based care management. It was recommended that interactive, sequential, disorder-specific treatment pathways be implemented to quickly provide clinicians with patient clinical status, treatment history, and decision support.

CONCLUSIONS: Specific informatics strategies have the potential to improve care for chronic illness. Software to implement these strategies should be developed, and rigorously evaluated within the context of organizational efforts to improve care.

KEY WORDS: chronic disease; informatics; clinical information systems; quality improvement; decision making.

J Gen Intern Med 22(Suppl 3):425-30

DOI: $10.1007 /$ s11606-007-0303-4

(C) Society of General Internal Medicine 2007

\section{BACKGROUND AND OBJECTIVES}

Chronic illnesses are a high priority for health care systems because they are costly, complex to manage, and result in substantial disability. Chronic illness is extremely common: Almost half of all Americans, more than 100 million people, live with a chronic condition. Half of those affected, more than 50 million, are living with more than one chronic illness. Costs for chronic illness care are approximately $80 \%$ of total health care costs, and rising. ${ }^{1}$ Despite these expenditures, the quality of care for chronic illness is often poor. ${ }^{2,3}$ Treatments known to be beneficial are provided only about $50 \%$ of the time, and inappropriate treatments are provided as much as $30 \%$ of the time. ${ }^{4}$ One problem is that health care organizations often utilize care models that respond well to acute problems, but are poorly designed for chronic illness care. In prevailing care models, clinicians have to focus brief visits on acute problems, crowding out time to attend to chronic disorders. Follow-up care is poorly coordinated, communication breakdowns are common, and patients often lack key skills and information they need to self-manage their illnesses. ${ }^{1,5,6}$

One leading approach to improving care for chronic illness is to implement "collaborative" or "chronic care" models. ${ }^{5,7,8}$ 
These care models use a longitudinal, team-based approach, and reorganize care to improve collaboration between an informed, activated patient and a prepared, proactive care team. Chronic care models that integrate patient information, clinical care management, and evidence-based treatments have been shown to improve patient outcomes. ${ }^{9}$ However, there are formidable obstacles to implementing these care models, and their use has been limited. Improving information flow, both between patient and provider and among providers, is a prerequisite, but the complexity of transmitting knowledge and patient information within the treatment environment has been a challenge. ${ }^{10,11}$ Health information technologies (HIT), such as electronic medical records (EMRs), can efficiently improve information flow. ${ }^{12,13}$ Indeed, HIT is specified as critical to implementation of many chronic care models. ${ }^{14}$ Although most physicians in the United States still do not use comprehensive EMRs, EMRs are now common in large provider organizations and many smaller organizations have adopted focused informatics tools. ${ }^{15-17}$ Whereas current HIT systems are often organized similar to paper charts, the diversity of potential systems is enormous. This has contributed to considerable uncertainty regarding which specific informatics components and strategies improve chronic illness care, slowing development and implementation efforts.

There has been extensive practical experience with HIT. ${ }^{18-21}$ Two relevant domains of HIT in chronic illness care are EMRs and personal health records (PHRs). EMRs have been developed to support the operations of health care providers, whereas PHRs provide services to patients and help them manage their health care data and illnesses. ${ }^{22,23}$ Although research on PHRs is limited, there is substantial research on EMRs. Recently, a committee of the Institute of Medicine (IOM) identified eight domains of care delivery that EMR systems should perform: health information/data, results management, order entry, decision support, communication and connectivity, patient support, administrative process, reporting, and population health. ${ }^{22}$ Despite an extensive body of research, in practice, EMR systems can be limited in their scope, or become complex and unwieldy. ${ }^{24}$ Also, prevailing systems often lack functions that support chronic illness care, such as disease registries, the capability to monitor panels of patients, facilitation of team communication, execution of treatment plans across primary and specialty care lines, and transition from text notes to computable fields.

This manuscript presents results from "Creating Health Informatics Applications for Collaborative Care" (CHIACC), a project to increase consensus regarding informatics technologies that support improvement in chronic illness care. Primary funding for CHIACC was provided by the Department of Veterans Affairs (VA). The VA operates the largest integrated health care system in the United States-a system with a comprehensive, fully computerized EMR. ${ }^{18,25,26}$ The goals of CHIACC were to understand how informatics can support improvement in the quality of care and to inform next steps in software development for organizations that have moved or are moving from paper-based to electronic records.

\section{METHODS}

The project consisted of four linked phases: (1) establish a steering committee to guide the project and discuss potential informatics components and strategies, (2) review the literature on informatics that could improve chronic illness care, (3) develop use cases that exemplify the complexities of chronic illness care, and (4) increase expert consensus regarding informatics components and strategies, taking into account the strengths and weaknesses of software previously developed, used, and evaluated. See Table 1 for the project's timeline.

The CHIACC project was guided by a 10-member multidisciplinary steering committee that had broad representation from VA and non-VA stakeholders in a variety of medical specialties and information systems. Members included physician experts in chronic diseases such as hypertension and depression, and individuals with expertise in improving the quality of care and information systems. The steering committee was tasked with ensuring that the project was consistent with national directions in improving care for chronic illness, and with prevailing information systems, including the VA national EMR.

Semistructured interviews were conducted with steering committee members to identify potential informatics components. The committee decided to adopt the eight IOM informatics functional domains ${ }^{22}$ and to study whether these

Table 1. CHIACC Study Timeline

\begin{tabular}{|c|c|c|}
\hline Date & Occurrence & Tasks \\
\hline October 2004 & $\begin{array}{l}\text { Steering Committee } \\
\text { meets in person }\end{array}$ & $\begin{array}{l}\text { Present study goals and } \\
\text { timeline. Solicit initial } \\
\text { ideas regarding } \\
\text { software components } \\
\text { and expert panel } \\
\text { composition. Establish } \\
\text { directions and priorities. }\end{array}$ \\
\hline $\begin{array}{l}\text { January- } \\
\text { February } 2005\end{array}$ & $\begin{array}{l}\text { Steering Committee } \\
\text { individual } \\
\text { interviews by } \\
\text { phone }\end{array}$ & $\begin{array}{l}\text { Elicit potential domains } \\
\text { to be included in } \\
\text { software. Elicit } \\
\text { strategies for having the } \\
\text { expert panel rate } \\
\text { domains. }\end{array}$ \\
\hline February 2005 & $\begin{array}{l}\text { Steering Committee } \\
\text { meets by phone }\end{array}$ & $\begin{array}{l}\text { Provide summary of } \\
\text { individual interviews } \\
\text { and finalize expert } \\
\text { panel membership. }\end{array}$ \\
\hline $\begin{array}{l}\text { Started March } \\
\text { 2005; published } \\
\text { April } 2007\end{array}$ & Literature review & $\begin{array}{l}\text { Characterize information } \\
\text { systems components } \\
\text { important in } \\
\text { supporting improved } \\
\text { care of chronic illness. }\end{array}$ \\
\hline June 2005 & $\begin{array}{l}\text { Use case } \\
\text { development }\end{array}$ & $\begin{array}{l}\text { Develop use } \\
\text { cases for depression, } \\
\text { schizophrenia, diabetes, } \\
\text { and comorbid disorders. }\end{array}$ \\
\hline July-August 2005 & $\begin{array}{l}\text { Expert panel } \\
\text { communication } \\
\text { via email }\end{array}$ & $\begin{array}{l}\text { Present summary of } \\
\text { literature review and } \\
\text { draft use cases. Expert } \\
\text { panel sends revisions. } \\
\text { Use cases revised. }\end{array}$ \\
\hline September 2005 & $\begin{array}{l}\text { Expert panel meets } \\
\text { in person }\end{array}$ & $\begin{array}{l}\text { Present full literature } \\
\text { review results. Present } \\
\text { revised use cases. } \\
\text { Increase consensus on } \\
\text { software components } \\
\text { and functions. }\end{array}$ \\
\hline October 2005 & Use cases finalized & $\begin{array}{l}\text { Revise and finalize use } \\
\text { cases based on expert } \\
\text { panel discussions. }\end{array}$ \\
\hline July 2006 & $\begin{array}{l}\text { Steering Committee } \\
\text { meets by phone }\end{array}$ & $\begin{array}{l}\text { Present expert panel } \\
\text { consensus, and finalize } \\
\text { use cases. }\end{array}$ \\
\hline
\end{tabular}


domains wholly captured software components that improve chronic illness care. The steering committee members gave feedback on the utility of each of the IOM domains and suggested additional functions they felt were important to the system within each domain. For example, the IOM domain of "decision support" includes computerized reminders and prompts, computer-assisted diagnosis, and access to treatment guidelines. The committee could add, "real time interpretation of patient unmet needs" as critical and fitting within this domain.

A systematic review of the literature was undertaken, covering studies evaluating information systems used in chronic illness care. ${ }^{19}$ The review searched the literature from 1996-2005. The primary goal was to understand which informatics components support best practices and which have the highest likelihood of successful implementation.

Building on the findings of the steering committee interviews and literature review, "use case models" were then developed for depression, schizophrenia, diabetes, and comorbid disorders. These disorders were chosen because of their high priority nationally, their fit with project objectives, and to include attention to comorbidity, which is the norm in chronic illness. The VA has a national Quality Enhancement Research Initiative (QUERI) program, which is designed to translate research discoveries into better patient care and systems improvements. ${ }^{27}$ There are nine QUERI programs, each focused on high-risk or highly prevalent disorders. For each use case, a workgroup was convened, with leadership from CHIACC and the relevant QUERI programs (i.e., mental health and diabetes). Each workgroup drew on results from the literature review and expert interviews, and on the clinical and research experience of the relevant QUERI program, in constructing their use case. Each use case was reviewed by a diverse group of clinicians from the field. This process resulted in draft use cases that became a foundation for the expert panel discussion, and began an iterative process of use case development.

An expert panel was formed to increase consensus regarding informatics system needs for improving chronic care. Consensus was necessary given (1) the literature review did not result in clear direction for software development and (2) the domains and function of software modules needed to be prioritized with the goal of serving multiple comorbid conditions. The panel process used a modified Delphi approach, and built consensus using iterative feedback on each proposed use case. $^{28,29}$ On any given informatics component, we sought agreement among the vast majority of panelists, but did not insist on complete agreement from all panelists. The composition of the expert panel included clinicians treating diabetes, depression, schizophrenia, and comorbid conditions; quality improvement and disease management experts; information system developers; and health care managers and policymakers. Experts were drawn from VA, the Substance Abuse and Mental Health Services Administration, and the health care industry. Diverse experience was important because a goal was to consider software modules that are consistent with EMRs and informatics developments nationally.

Before the expert panel meeting, panel members were provided with results from the literature review and the initial use cases. The panel provided feedback on the use cases, and a second version of the use cases were made based on this feedback. At the in-person expert panel meeting in the fall of
2005, the second version of the use case models were presented and used to guide a discussion regarding requirements of informatics modules to support improvement in chronic illness care. Diabetes, depression, schizophrenia, and comorbidity were each discussed separately, with each discussion led by a clinician researcher expert in the disorder. Detailed minutes were taken by two research assistants. At the close of discussion of each disorder, a final list of software components was agreed upon and prioritized by the group. After all diseases had been discussed, the lists for each disease were combined and reprioritized for a final list of priorities for software components to address chronic illness generally. After the expert panel meeting, the lists for each disease were used to revise the use case models into a final version that could be used to inform software development.

Use Cases. The use case methodology is an iterative process often used in HIT and other informatics development. ${ }^{30,31}$ The goal is to capture the requirements inherent in a system's response to primary actors. Whereas there are various ways to document a use case, a common approach is to write a structured story that describes how the informatics systems assist in certain behaviors. A use case describes system functionality and is referred to at all stages in the software development process: programmers create a software prototype based on the use case, developers then review each successive prototype for conformance to the use case, and field testing ensures that the prototype correctly implements the use case. Whereas this project also developed use cases for depression and schizophrenia, the diabetes use case is discussed here in detail because it provides the best example of chronic illness care that includes comorbidity.

A use case begins with a vision for the behaviors described. For CHIACC, the vision was to improve the quality of care through implementation of guideline-concordant treatments. All the actors involved in the case are listed. In diabetes these were the primary care provider, clinical care manager, specialists who provide consultation, and the patient. The trigger that activates the use case is described, and the flow of behaviors and events that follow from that trigger are outlined, including various optional conditions. In the diabetes use case, a trigger occurs when a primary care provider diagnoses diabetes and refers a patient to care management for initial assessment, education, and follow-up. Another trigger to activate the use case would occur when a care manager identifies a patient at high risk for diabetes in need of more intensive education, treatment, or monitoring and initiates a visit or phone call with the patient. Then a typical course of events follows, with highlighting of areas where HIT can provide improved care of quality. In diabetes, the use case describes separate visits with the medical assistant, the primary care provider, and the care manager, and the resulting communication among this team and with the patient. In addition, there is information about tracking the patient's progress and population management. The use case illustrates how HIT can be used to support quality care through components such as a comprehensive care plan, lab value and medication alerts, improved provider communication, and tracking of patient outcomes. The use case provides alternative pathways when, for example, a patient is particularly noncompliant or especially self-motivated and activated. 


\section{RESULTS}

Steering Committee interviews revealed that the IOM domains were useful but varied in importance. The domain rated lowest in utility was "administrative processes" and the highest was "health information and data." The Steering Committee added a number of additional features to the IT domains, with each domain acquiring several additions overall. For example, the IOM defines "health information and data" as, "electronic storage and immediate access to diagnoses, allergies, lab test results, medications, clinical notes, demographics." Committee members added several items, including patient assessment and surveys, routine outcomes assessments, and results of ongoing assessments.

Results from the literature review have been published. ${ }^{19}$ Much of the literature is nonexperimental. In studies with experimental designs, components related to positive outcomes are connection to an EMR, computerized prompts, population management, specialized decision support, electronic scheduling, and patient health records. The only informatics component not associated with improved outcomes was access to treatment guidelines. This is consistent with some previous, broader reviews. ${ }^{21,32}$

The expert panel agreed that information systems should be patient-centered and emphasize patient preferences, functioning, and quality of life. The "recovery" model, which was originally developed for mental health, ${ }^{33}$ was seen as applicable to other chronic medical disorders. This recovery model emphasizes the importance of moving beyond disease alone to strategies for supporting individuals with chronic illness as they work toward life goals and aspirations. ${ }^{34}$

The panel agreed that the routine assessment of critical outcomes was necessary, using reliable, valid, and commonly used measures such as the nine-item scale of the patient health questionnaire for depression or blood pressure for hypertension. ${ }^{35}$ Choice of outcomes should be guided by quality improvement priorities. The panel recommended using automatic scoring algorithms to link problematic outcomes scores to appropriate treatment recommendations and agreed that these results should be easily accessible and interpretable by providers at the time of the clinical encounter. There were several ideas for displaying these results, such as a timeline graphing scores against medication trials, clinical encounters, and landmark events. There have been published informatics tools that graph blood pressure against antihypertensive medications on a timeline. ${ }^{36}$ Outcomes data should also be usable for population-based improvement of health care quality.

A key problem with current EMRs is information overload, and data presentation that does not efficiently meet the needs of providers in complex cases. In response, the panel stated that there should be an interactive, sequential, and comprehensive treatment plan in the EMR. Each medical condition in the patient's problem list would be accompanied by a clinical care pathway, which could be used by clinicians to quickly identify the current stage of treatment for each of the patient's problems in relation to best-practice algorithms. For example, in the management of depression, the care pathway could start with initiation of either antidepressant medication or psychotherapy, then proceed to intensifying the treatment, and trying alternative or combination treatments for patients failing to respond. Once depression has remitted, the patient would enter a maintenance phase of treatment. The treatment plan would clearly indicate which phase of treatment the patient is in, and how long they had been there. Outcome indicators, such as depression severity, could be graphically displayed in relation to treatment trials.

With regard to the diabetes use case, panel members agreed that communication, collaboration, and coordination of care among all providers is critical to improving care. A method such as an electronic flow sheet to track steps in care over time would be beneficial to the coordination of diabetes care. Panel members believed that there is a need for scheduling long-term care needs (such as eye exams and foot care for diabetes) and that a tickler alert system would be ideal for long-term, automated scheduling.

Another recommendation was structured, automated templates for progress notes, allowing providers to easily report and access critical information regarding treatment history and planned treatment steps. The panel agreed that templates with functionalities such as guideline-based checklists for providers could enhance diabetes care. Other suggestions that were endorsed by the panel included creating provider alerts for gaps in prescription refills to target issues with medication adherence, and creating a system of automated phone messages for patients, reminding them of appointments and medication refills.

The panel concluded with a discussion of comorbid disorders. Panel members emphasized that the treatment plan should include each of the patients' medical conditions, whereas allowing for prioritization of problems. Accessing one medical condition, for example, diabetes, could trigger the display of other important and related comorbid conditions, such as hyperlipidemia, as well as the display of relevant labs and outcome measures. Furthermore, panel members believed that the treatment plan should list all providers and care managers associated with each condition and be shared across treatment sites. Because comorbidity is very common in diabetes care, the decision was made to merge the diabetes and comorbidity use cases. The use cases were revised based on results from the panel, and the final versions are available online (DOI 10.1007/s11606-007-0303-04).

\section{CONCLUSIONS}

The science of chronic illness care is rapidly advancing, and there is increasing pressure to improve the quality and efficiency of care for these disorders. There is also rapid evolution in health informatics tools that help improve chronic illness care. Because research on the effectiveness of specific informatics tools is inadequate to definitively characterize HIT needs, and will likely remain so, this project made use of a national expert panel process to increase consensus in this area. Whereas HIT can serve diverse agendas, the focus here was on HIT as a tool to improve the quality of care. The VA has one of the largest and most successful quality improvement systems in health care nationally, ${ }^{2}$ and this project drew heavily from that experience. With this focus, the expert panel demonstrated remarkably strong consensus. Given the prevalence of comorbidity, and the need to manage care over time, the panel recommended that an interactive, sequential, disorder-specific treatment planning pathway system be central to HIT development efforts. To do this, treatment 
algorithms will need to be implemented in software. Because informatics should be patient-centered and recovery-oriented, critical outcomes need to be routinely assessed and retrievably coded. With this approach, decision support systems can quickly show the clinician where the patient is in their longitudinal treatment plan, present the history of outcomes and treatment use, and provide guidance regarding current treatment options. Data can also be useful to managers and policymakers to characterize workflow and inform quality improvement efforts.

Successful use of HIT to improve chronic illness care requires attention to specific implementation issues and strategies. ${ }^{37,38}$ For example, many physicians in the US still do not use an EMR, and communication among the panoply of hospital and community providers' electronic systems is woefully inadequate. Whereas EMRs can be powerful tools to improve the quality of care, their implementation is neither easy nor cheap, and barriers are especially high for solo and small group providers. A recent review of HIT implementation concluded that developers need to consider structural, financial, policy-related, cultural, and organizational barriers to implementation and strategies to overcome them. ${ }^{11}$ Cost can be a barrier when HIT supports implementation of evidencebased practices. Many evidence-based strategies, such as the chronic care model, rely on care managers, nurses, and other nonphysician staff to provide ongoing, assertive management of disease. However, typical reimbursement mechanisms were not designed for this purpose, rendering implementation prohibitively expensive. HIT can provide some help in this regard, minimizing staff costs, detecting errors, and maximizing efficiency and coordination of care. ${ }^{14}$ Also, for organizations that have a staged approach to implementing HIT, software to support chronic illness care can be an early step in the process of EMR implementation. This can have an impact on quality with lower cost burden than a full EMR.

Much of the research on use of HIT is nonexperimental and relatively few studies have examined quality of care as an outcome. Substantially more research is needed, and, when possible, experimental designs should be employed. However, much of HIT development occurs outside the context of research studies, in support of specific projects or clinical missions. In these situations, it will be important to use robust formative research methods that include usability testing but also go beyond it to evaluate the role of information systems in clinical and policy efforts to improve care. ${ }^{39}$

In this project, the expert panel process resulted in the development of use cases for depression, schizophrenia, and diabetes with comorbidity. Because informatics development, usability testing, and the evaluation of informatics effectiveness are iterative processes, these use cases are not final versions but rather a step toward ongoing informatics development. Use cases will need to be modified as the science of quality improvement evolves, and as more research is done on pay for performance and other strategies. They will also need to be adapted to the needs of the specific clinicians, organizations, the patient populations they serve, and the quality improvement strategies that are used. There may also need to be modifications as PHRs become more widely used and studied. Further informatics development work will certainly be needed to extend EMRs and PHRs in new directions. Therefore, software needs to be flexible enough to support and incorporate new findings about how to improve care for patients.
CHIACC illustrated the value of bringing together policymakers as well as experts in implementation science and HIT to inform software development. To broadly improve chronic illness care, HIT must be consistent with organizational and population-based efforts to improve treatment quality, and meet the needs of clinicians and clinic staff. Implementation must be feasible. It is quite challenging to simultaneously meet the needs of the wide variety of HIT stakeholders, and there are numerous examples of HIT systems that have not had broad impact or that have failed to meet expectations. Informatics has the potential to substantially improve chronic illness care. To fully achieve this potential, HIT development should be integrated with a systematic approach to health care quality improvement and implementation science.

Acknowledgements: We would like to thank Nick Dewan, Allan Finkelstein, John Fortney, Denise Hynes, Katy Lysell, Susan McCutcheon, Jennifer Pope, Carol Simons, Jeff Smith, and the following members of the CHIACC expert panel:

Tom Craig, MD, VA Office of Quality and Performance

Eve Kerr, MD, VA Diabetes QUERI

Linda McIvor, MS, MHS, VA QUERI

Alec Miller, MD, University of Texas

Sarah Minden, MD, SAMHSA and Abt Associates

David Nace, MD, McKesson Health Solutions

Paul Nichol, MD, Seattle VA

Hank Rappaport, MD, VA Office of Information

Lisa Rubenstein, MD, Sepulveda VA HSR\&D Center of Excellence and RAND

Conflict of interest statement: Dr. Young, Dr. Chaney, Dr. Bonner, Dr. Cohen, Dr. Doebbling, Dr. Goldstein, Dr. Kerr, and Dr. Nichol as well as Ms. Shoai and Ms. Perrin are employees of the Department of Veterans Affairs. Dr. Chaney, Dr. Cohen, and Dr. Bonner and Ms. Shoai received salary support from the grant that funded this research. Other authors received support from grants involving related projects.

Corresponding Author: Alexander S. Young, MD, MSHS; West Los Angeles VA Healthcare Center, VA Desert Pacific Mental Illness Research, Education and Clinical Center (MIRECC), 11301 Wilshire Blvd. (210A), Los Angeles, CA 90073, USA (e-mail ayoung@ucla.edu).

\section{REFERENCES}

1. Partnership for Solutions: Johns Hopkins University for the Robert Wood Johnson Foundation. Chronic Conditions: Making the Case for Ongoing Care. Available at http://www.rwjf.org/research/researchdetail.jsp? id=1502\&ia=142; September 2004. Accessed December 4, 2006.

2. Asch SM, McGlynn EA, Hogan MM, et al. Comparison of quality of care for patients in the Veterans Health Administration and patients in a national sample. Ann Intern Med. 2004;141(12):938-45.

3. Lehman AF. Quality of care in mental health: the case of schizophrenia. Health Aff (Millwood, Va). 1999;18(5):52-65.

4. McGlynn EA, Asch SM, Adams $J$, et al. The quality of health care delivered to adults in the United States. N Engl J Med. 2003;348 (26):2635-45.

5. Bodenheimer T, Wagner EH, Grumbach $\mathbf{K}$. Improving primary care for patients with chronic illness. JAMA 2002;288(14):1775-9.

6. Wagner EH. Chronic disease management: what will it take to improve care for chronic illness? Eff Clin Pract. 1998;1(1):2-4.

7. Bodenheimer T, Wagner EH, Grumbach $\mathbf{K}$. Improving primary care for patients with chronic illness: the chronic care model, part 2. JAMA 2002;288(15):1909-14

8. Wagner EH, Austin BT, Von Korff M. Organizing care for patients with chronic illness. Milbank Q. 1996;74(4):511-44.

9. Bodenheimer T. Interventions to improve chronic illness care: evaluating their effectiveness. Dis Manag. 2003;6(2):63-71. 
10. Young AS, Klap R, Sherbourne CD, Wells KB. The quality of care for depressive and anxiety disorders in the United States. Arch Gen Psychiatry. 2001;58(1):55-61.

11. Doebbeling BN, Chou AF, Tierney WM. Priorities and strategies for the implementation of integrated informatics and communications technology to improve evidence-based practice. J Gen Intern Med. 2006;21 (suppl 2):S50-7.

12. Institute of Medicine. Crossing the Quality Chasm: A New Health System for the 21st Century. Washington, DC: National Academies Press; 2001.

13. Reid P, Compton W, Grossman J, Fanjiang G, eds. Building a better delivery system: a new engineering/health care partnership. Washington, DC: National Academies Press; 2005.

14. Hillestad R, Bigelow J, Bower A, et al. Can electronic medical record systems transform health care? Potential health benefits, savings, and costs. Health Aff (Millwood, Va). 2005;24(5):1103-17.

15. Bower AG. The Diffusion and Value of Healthcare Information Technology. Santa Monica: RAND; 2005 (report no. M6-272-HLTH)

16. Burt CW, Sisk JE. Which physicians and practices are using electronic medical records? Health Aff (Millwood, Va). 2005;24(5):1334-43.

17. Gans D, Kralewski J, Hammons T, Dowd B. Medical groups' adoption of electronic health records and information systems. Health Aff (Millwood, Va). 2005;24(5):1323-33.

18. Perlin JB, Kolodner RM, Roswell RH. The Veterans Health Administration: quality, value, accountability, and information as transforming strategies for patient-centered care. Am J Manag Care. 2004;10(11 Pt 2):828-36.

19. Dorr D, Bonner LM, Cohen AN, et al. Informatics systems to promote improved care for chronic illness: a literature review. J Am Med Inform Assoc. 2007;14(2):156-63.

20. Andrus RW. Integrating a clinical system. In: Kolodner R, ed. Computerizing Large Integrated Health Networks: The VA Success. Berlin Heidelberg New York: Springer; 1997.

21. Chaudhry B, Wang J, Wu S, et al. Systematic review: impact of health information technology on quality, efficiency, and costs of medical care. Ann Intern Med. 2006;144(10):742-52.

22. Institute of Medicine. Key Capabilities of an Electronic Health Record System. Washington, DC: National Academies Press; 2003.

23. Department of Veterans Affairs. MyHealtheVet History. Available at https://www.myhealth.va.gov/mhvPortal 2005. Accessed December 5, 2006.

24. Lyons SS, Tripp-Reimer T, Sorofman BA, et al. VA QUERI informatics paper: information technology for clinical guideline implementation: perceptions of multidisciplinary stakeholders. J Am Med Inform Assoc 2005;12(1):64-71.

25. Bernstein SJ. VA health services research: lessons for the world's healthcare organizations. Am J Manag Care 2004;10(11 Pt 2):825-7.

26. Kupersmith J, Francis J, Kerr E, et al. Advancing evidence-based care for diabetes: lessons from the Veterans Health Administration. Health Aff (Millwood, Va). 2007;26(2):w156-68.

27. Hagedorn $\mathbf{H}$, Hogan $\mathbf{M}$, Smith $\mathbf{J L}$, et al. Lessons learned about implementing research evidence into clinical practice. Experiences from VA QUERI. J Gen Intern Med. 2006;21(suppl 2):S21-4.

28. Young AS, Forquer SL, Tran A, Starzynski M, Shatkin J. Identifying clinical competencies that support rehabilitation and empowerment in individuals with severe mental illness. J Behav Health Serv Res. 2000;27 (3):321-33.

29. Fitch K, Bernstein S, Aguilar MD, et al. RAND/UCLA Appropriateness Method User's Manual. Santa Monica, Calif: RAND; 2001.

30. Cockburn A. Writing Effective Use Cases. New York: Addison-Wesley; 2001

31. Bittner K, Spence I. Use Case Modeling. New York: Addison-Wesley; 2003.

32. Kawamoto K, Houlihan CA, Balas EA, Lobach DF. Improving clinical practice using clinical decision support systems: a systematic review of trials to identify features critical to success. BMJ. 2005;330(7494):765.

33. President's New Freedom Commission on Mental Health. Achieving the Promise: Transforming Mental Health Care in America. Available at: http://www.mentalhealthcommission.gov/reports/reports.htm; 2003. Accessed April 30, 2007.

34. Mueser KT, Corrigan PW, Hilton DW, et al. Illness management and recovery: a review of the research. Psychiatr Serv. 2002;53(10):1272-84.

35. Young AS, Mintz J, Cohen AN. Using information systems to improve care for persons with schizophrenia. Psychiatr Serv. 2004;55(3):253-5.

36. Goldstein MK, Hoffman BB. Graphical displays to improve guidelinebased therapy of hypertension. In: Izzo J, Black H, eds. Hypertension Primer. Baltimore: Williams \& Wilkins; 2003.

37. BootsMiller BJ, Yankey JW, Flach SD, et al. Classifying the effectiveness of Veterans Affairs guideline implementation approaches. Am J Med Qual. 2004;19(6):248-54.

38. Bates DW, Kuperman GJ, Wang S, et al. Ten commandments for effective clinical decision support: making the practice of evidence-based medicine a reality. J Am Med Inform Assoc. 2003;10(6):523-30.

39. Stetler CB, Legro MW, Wallace CM, et al. The role of formative evaluation in implementation research and the QUERI experience. J Gen Intern Med. 2006;21(suppl 2):S1-8. 